\title{
Periodicities of the RV Tauri-type pulsating star DF Cygni: A combination of Kepler data with ground-based observations
}

\author{
A. Bódi ${ }^{1}$, K. Szatmáry ${ }^{1}$, and L. L. Kiss ${ }^{2,3}$ \\ 1 Department of Experimental Physics and Astronomical Observatory, University of Szeged, 6720 Szeged, Dóm tér 9, Hungary \\ e-mail: abodi@titan.physx.u-szeged.hu \\ 2 Konkoly Observatory, Research Centre for Astronomy and Earth Sciences, Hungarian Academy of Sciences, 1121 Budapest, \\ Konkoly Thege M. út 15-17, Hungary \\ 3 Sydney Institute for Astronomy, School of Physics A28, University of Sydney, NSW 2006, Australia
}

Received 15 July 2016 / Accepted 22 September 2016

\begin{abstract}
Context. The RV Tauri stars constitute a small group of classical pulsating stars with some dozen known members in the Milky Way. The light variation is caused predominantly by pulsations, but these alone do not explain the full complexity of light curves. High-quality photometry of RV Tau-type stars is very rare. DF Cygni is the only member of this class of stars in the original Kepler field, hence allowing the most accurate photometric investigation of any RV Tauri star to date.

Aims. The main goal is to analyse the periodicities of the RV Tauri-type star DF Cygni by combining four years of high-quality Kepler photometry with almost half a century of visual data collected by the American Association of Variable Star Observers (AAVSO).

Methods. Kepler quarters of data were stitched together to minimize the systematic effects of space data. The mean levels have been matched with AAVSO visual data. Both datasets were submitted to Fourier and wavelet analyses, while the stability of the main pulsations was studied with the $\mathrm{O}-\mathrm{C}$ method and analysis of time-dependent amplitudes.

Results. DF Cygni shows very rich behaviour on all timescales. The slow variation has a period of $779.606 \mathrm{~d}$ and it has been remarkably coherent during the whole time span of the combined data. On top of the long-term cycles, the pulsations appear with a period of $24.925 \mathrm{~d}$, or the double period of $49.85 \mathrm{~d}$ if we take the RV Tau-type alternation of the cycles into account. Both types of light variation significantly fluctuate in time, with a constantly changing interplay of amplitude and phase modulations. Long-period change (i.e. the RVb signature) somewhat resembles the long secondary period (LSP) phenomenon of pulsating red giants, whereas short-period pulsations are very similar to those of the Cepheid variables. Comparing the pulsation patterns with the latest models of Type-II Cepheids, we found evidence of strong non-linear effects that are directly observable in the Kepler light curve.
\end{abstract}

Key words. stars: AGB and post-AGB - stars: variables: general - stars: oscillations

\section{Introduction}

RV Tauri stars are evolved low-mass F, G, and K-type pulsating supergiants that are located above Population II Cepheids in the instability strip. Traditionally, they are classified into two subgroups. The RVa type is characterized by alternating minima with typical periods longer than 20 days. The RVb stars show an additional long-term variation in the mean brightness, with typical periods of 700-1200 days. The short-period variation is interpreted with fundamental mode pulsation, while the long-term phenomenon is commonly interpreted as caused by a periodic obscuration of a binary system by a circumbinary dust disk (Lloyd Evans 1985; Pollard et al. 1996; Van Winckel et al. 1999; Fokin 2001; Maas et al. 2002; Gezer et al. 2015).

RV Tauri stars have similar luminosities, but higher effective temperatures than Miras. The luminosity function of RV Tauri stars mainly overlaps with the low-luminosity portion of the Mira luminosity function. There are similarities between their observational characteristics. A few Miras show double maxima, which is a feature that is common in RV Tauri stars (e.g. $\mathrm{R}$ Cen, R Nor). Some Miras and semi-regular variables may exhibit the same (or physically similar) quasi-periodic, long-term mean brightness variations as RVb stars (e.g. RU Vir; Willson \& Templeton 2009). Also, there is extensive literature on the so-called long secondary periods of asymptotic giant branch stars, which is a phenomenon that still puzzles researchers (Wood et al. 1999; Takayama et al. 2015) and causes light curves that are surprisingly similar to those of RVb stars.

Originally, RV Tauri stars were classified as Cepheid variables because of their similarities and the poor quality of their light curves. Type II Cepheids may be divided into groups by period, such that the stars with periods between 1 and 5 days (BL Her class), 10-20 days (W Vir class), and greater than 20 days (RV Tauri class) have different evolutionary histories (Wallerstein 2002). In each case, the shape of the light curve is nearly sinusoidal, but the RV Tau-type stars show alternating minima, which means that every second minimum is shallower. The irregularity of the minima grows as the period becomes longer. The light curve of some RV Tau-type stars randomly switches into a low-amplitude irregular variation and then switches back into the previous state (e.g. AC Her, Kolláth et al. 1998; and R Sct, Kolláth 1990; Buchler et al. 1996). RV Tauri variables were placed among the post-AGB stars (Jura 1986), which are rapidly evolving descendants of stars with initial masses lower than $8 M_{\odot}$. In the late stages of their evolution they cross the Hertzsprung-Russell diagram (HRD) from the cool asymptotic giant branch (AGB) to the ionizing temperature 
of planetary nebula nuclei. During this process they cross the classical instability strip, in which large-amplitude radial oscillations are driven by the $\kappa$ mechanism, while the complexity of the post-AGB variability pattern depends on their location in the HRD relative to the classical instability strip (Kiss et al. 2007).

Several models attempted to reproduce the light variations of RV Tauri stars. The short period variation can be easily explained by summing two sine curves if the ratios of their frequencies are $1: 2$ and the phase difference is $\pi / 2$ (Pollard et al. 1996). Buchler et al. (1996) and Kolláth et al. (1998) established a more sophisticated model to describe the light curve of R Sct and AC Her via low-dimensional chaos. Owing to several phenomena that occur in the light curves and spectra of RVb stars, it is difficult to explain the typical long-term variation. A long-term photometric and spectroscopic survey was carried out by Pollard et al. (1996, 1997). They revealed that in some RVb stars, the reddest colours occur slightly after long-term light minimum. Furthermore, the light and colour amplitude of the short-term period is smaller during the long-term minima. These authors found that the equivalent width of the $\mathrm{H} \alpha$ emission lines in the spectrum vary with the phase of the long-term period. They concluded that the damping of the pulsation amplitude is difficult to explained with the popular model of light variation of RVb stars by a binary system that is periodically obscured by circumstellar or circumbinary dust disk (Percy 1993; Waelkens \& Waters 1993; Willson \& Templeton 2009). They also concluded that the $\mathrm{H} \alpha$ emission is caused by passing shock waves throughout the stellar photosphere. Pollard et al. (2006) proposed a possible dust-eclipse model whose arrangement can explain both the photometric and spectroscopic characteristics of the long-term phenomena. Unfortunately, the quality of the measurements did not allow us to investigate the pulsation mechanism in detail, except in the case of R Sct and AC Her.

Gezer et al. (2015) studied the spectral energy distribution (SED) of Galactic RV Tauri stars based on WISE infrared photometry. The objects with circumstellar disks have near-IR excess in the SED. The light curve of the members of this group shows variable mean magnitude, while there is a clear correlation between disk sources and binarity. Consequently, binarity is connected to the long-term changes of the mean brightness. On the other hand, both Gezer et al. (2015) and Giridhar et al. (2005) investigated the photospheric chemical anomaly called depletion as a sign of dust-gas separation. Two scenarios were proposed for objects with anomalous abundances: first, single stars with dust-gas separation in their stellar wind and, second, binary stars in which dust-gas separation is present in a circumbinary disk (Giridhar et al. 2005). The latter is consistent with the RVb binary hypothesis. Gezer et al. (2015) found that the presence of a disk seems to be a necessary but not sufficient condition for the depletion process to become efficient.

The subject of this paper, the bright RVb-type variable $\left(V_{\max } \approx 10.5 \mathrm{mag}, V_{\min } \approx 13 \mathrm{mag}\right.$ ) DF Cygni, was discovered by Harwood (1927). The period was found to be 49.4 days between the principal minima. Some years later a long period of $790 \pm 10$ days was found (Harwood 1936, 1937). The period of the radial pulsation is about 50 days, and the long secondary period is about 775 days (Percy 2006). The spectral and colour variation was investigated by Preston et al. (1963) who found $\mathrm{CN}$ bands in the spectrum. Gezer et al. (2015), in their search for disk sources, labelled DF Cygni as uncertain based on its SED, while the star only appears as marginally depleted, if at all (Giridhar et al. 2005; Gezer et al. 2015). Putting everything together, DF Cygni can be considered as one of the better known
RV Tauri-type variable stars, and the only member of this class in the original Kepler field.

Here we present a detailed light curve analysis of DF Cygni by combining about $48 \mathrm{yr}$ of visual observations with the ultraprecise space photometry from Kepler with a time span of about $4 \mathrm{yr}$. The two sources of the data permit investigations both with very high frequency resolution and with extreme photometric accuracy at least over two cycles of the RVb variability. In Sect. 2 we describe the data sources and the preparation of the sets before the analyses. The details of the light curve analysis are presented in Sect. 3, with the discussion of results in Sect. 4. A brief summary is given in Sect. 5.

\section{Preparation of the light curves}

The light curve data collected by the American Association of Variable Star Observers (AAVSO) were downloaded from the public website of the AAVSO ${ }^{1}$. From the available types of measurements only the longest dataset, corresponding to the almost five decades of visual brightness estimates, was used. In total, there were 5924 individual visual magnitudes from over 110 amateur astronomers, obtained between October 1968 and July 2015. Their typical precision is of the order of $\pm 0.3 \mathrm{mag}$ (Kiss et al. 1999), so that the next step was to calculate five-day bins. Larger bin size would have smoothed out too much of the short-period variation, while shorter bins would contain too few data points to average out the observational errors. On average, there are 10-15 points per bin, so that the estimated accuracy of the binned points is about $\pm 0.1 \mathrm{mag}$.

The Kepler dataset was downloaded from KASOC database, which is maintained by the Kepler Asteroseismic Science Consortium (KASC). The original data include, among other control parameters, the Barycentric Julian Date, the raw and corrected Kepler fluxes and the estimated uncertainties (which were of the order of $70 \mathrm{ppm}$ per single observation). DF Cygni, as one of the long-cadence (one point per every $29.4 \mathrm{~min}$ and short gaps between the quarters) KASC targets, was observed by Kepler throughout the entire more than four years (17 quarters) of operations in the original field. Since Kepler rolled 90 deg every quarter of the year, the image of DF Cygni fell on different CCD chips each quarter and hence systematic shifts occurred from quarter to quarter. Given that the length of a quarter and the dominant variability timescale are of the same order, there is a great difficulty in distinguishing the quarter-to-quarter variations from the intrinsic stellar variability.

In our study we followed the same approach as Bányai et al. (2013), who used short segments of the data immediately before and after the gaps between subsequent quarters to stitch the quarters by producing the most continuous curve. To accomplish this, Kepler fluxes were converted to magnitudes and only vertical shifts were allowed when joining the data from quarter to quarter. In a very small number of cases, further tiny corrections had to be carried out manually to reach the most satisfactory continuity with no apparent jumps.

The final light curves of DF Cygni are plotted in Fig. 1. Here the small red diamonds show the five-day means of the AAVSO visual data and the blue line refers to the corrected Kepler curve. The zero-point of the stitched Kepler data was matched with the simultaneous visual light curve so that the two curves overlap as much as possible. Also, the subsets shown in the individual panels in Fig. 1 were deliberately selected to cover exactly two cycles of the $\mathrm{RVb}$ variability ( 1570 days $=4.3 \mathrm{yr} / \mathrm{panel}$ ).

\footnotetext{
http: //WWw . aavso.org
} 


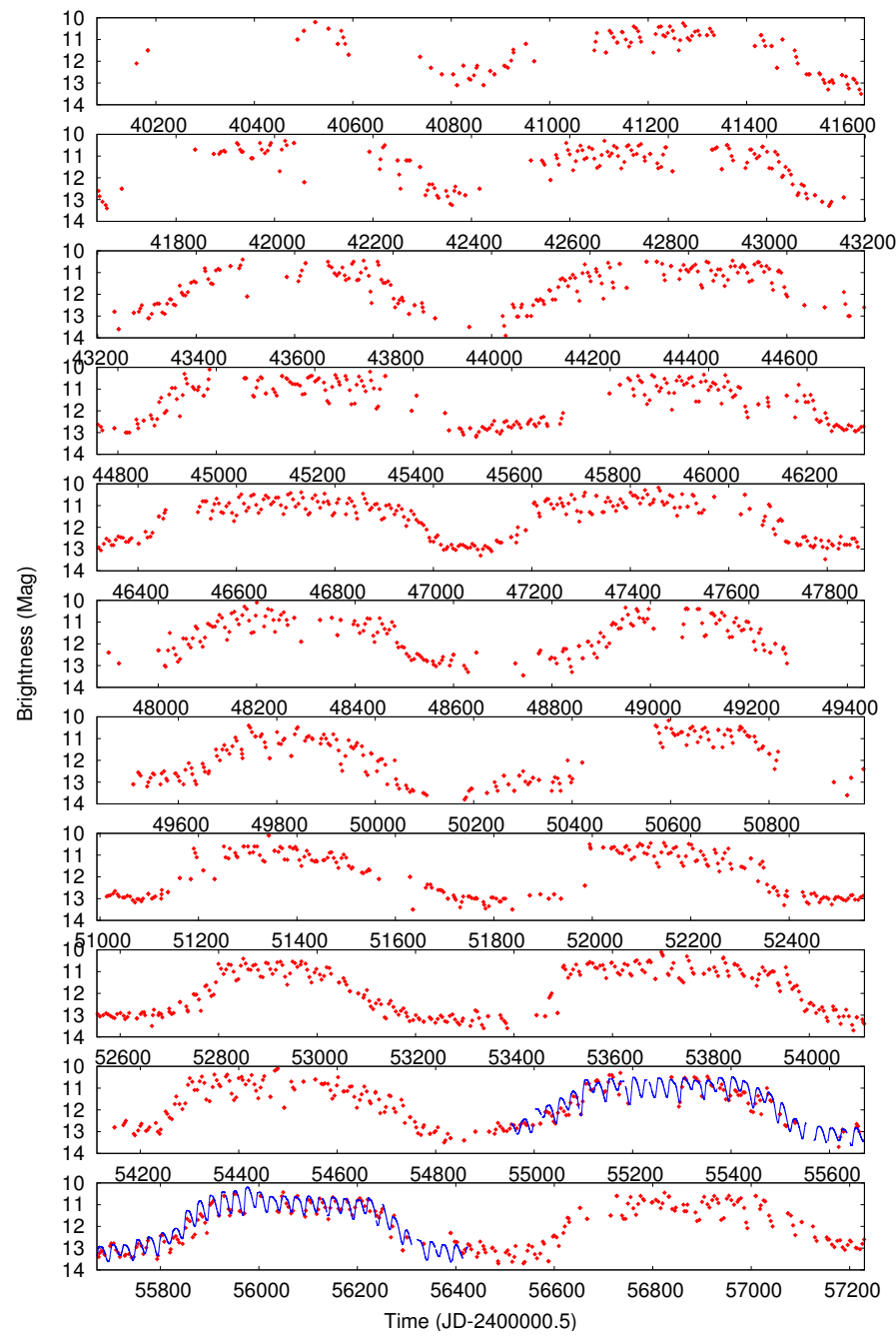

Fig. 1. Light curves of DF Cygni via AAVSO (red dots, 5-day means) and Kepler (blue line).

It is already apparent from Fig. 1 that the long-period cycles were coherent during the whole $48 \mathrm{yr}$ of visual observations. There were slight changes in the shape of the cycles, such as the sometimes flat-shaped maxima, but the faintest states were repeated very accurately with no apparent phase shift. On the other hand, the short-period variability changed a lot both in amplitude and in the shape of the cycles. The information on the latter is however very limited given that the five-days binning results in approximately 10 points per full RV Tau cycle. The fine details of pulsations are only visible in the continuous Kepler light curve. Nevertheless, we analyzed the two light curves in a similar fashion with all methods, so that we can directly compare their informational content.

\section{Light curve analysis}

We analysed the data with the traditional methods of Fourier analysis, O-C diagram, and wavelet analysis. Some of these were directly applicable to the original data, while some needed further processing steps, such as removing the long-term variation of the mean brightness. All the frequency spectra and then individual frequencies, amplitude, phase values, and their uncertainties were calculated with the Period04 software of Lenz $\&$ Breger (2005), using the least squares method when the

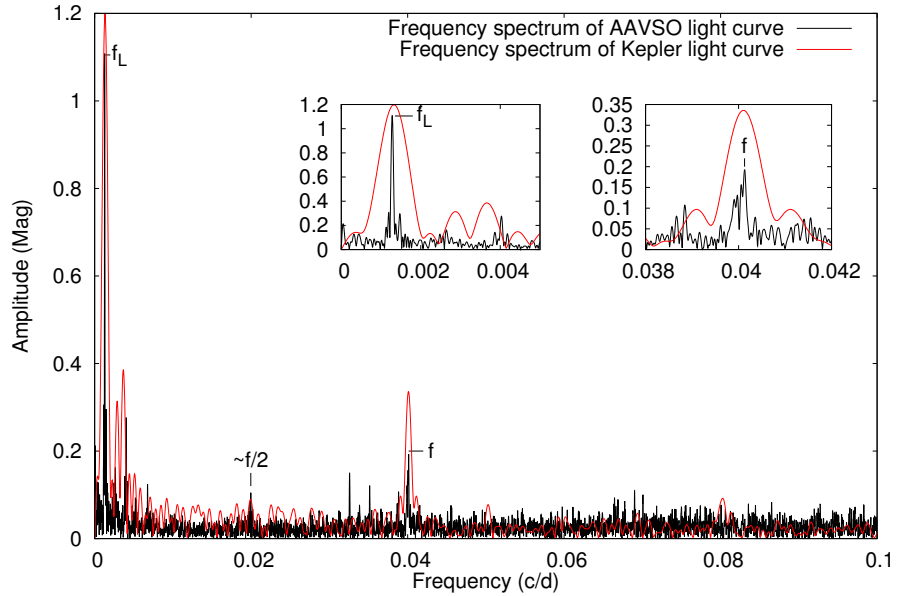

Fig. 2. Frequency spectra from the AAVSO (black) and Kepler (red) light curves of DF Cygni. Two zooms to $f_{\mathrm{L}}$ and $f$ are shown in the inserts.

maximum number of iterations were not reached. The signalto-noise ratio for each peak in the frequency spectrum was measured from the mean local amplitude value in the surrounding region ("noise"). The wavelet map was generated by the Fortran code WWZ of Foster (1996) where the decay parameter " $c$ " was set to 0.0125 . This value is appropriate when the timescale of variability ranges from tens of days to hundreds of days. Details of the time-frequency distribution were extracted with selfdeveloped scripts, which revealed specific information on the stability of the frequency peaks and their amplitudes.

The AAVSO visual light curve begins in 1968 and now covers 22 long-term cycles. The full peak-to-peak amplitude is about $3.45 \mathrm{mag}$. The long-term maxima are flat with similar brightnesses, but different durations. The minima are mostly shorter and curved, showing flat portions with a duration of approximately 100-200 days. The shape of the short-term variation can hardly be recognized in the AAVSO light curve, but the accuracy of the Kepler measurement allows us to analyse the 59 shortterm cycles covered from space. The alternation of the minima is clearly visible as are the variations of the maxima, a behaviour that is largely hidden in the visual dataset.

The frequency spectra of the two datasets show two significant peaks corresponding to the long-term (labelled as $f_{\mathrm{L}}$ ) and the short-term (labelled as $f$ ) variations (see Fig. 2 and Table 1). The $f / 2$ is also significant in the AAVSO spectrum, but nothing else is above the noise threshold. While the widths of the peaks are different, as expected for the different time-span $T_{\text {obs }}$ (the frequency resolution scales with $1 / T_{\text {obs }}$ ), it is evident that $f_{\mathrm{L}}$ was much more coherent in the last five decades than $f$. The latter peak at around $0.04 c / d(P \sim 25 \mathrm{~d})$ is split in the AAVSO data; this split is caused by the temporal changes of the frequency or the phase. The derived $f_{\mathrm{L}}$ and $f$ values are in good agreement if we compare the AAVSO and Kepler data, but the AAVSO set enables a more accurate frequency determination for $f_{\mathrm{L}}$, because of the much longer time span of the light curve. We note that all the additional peaks in the frequency spectrum of the AAVSO data correspond to the yearly or monthly aliases of three dominant peaks $\left(f_{\mathrm{L}}, f\right.$, and $\left.f / 2\right)$.

The Kepler spectrum displays a significantly richer frequency content. After pre-whitening with $f_{\mathrm{L}}$, the residual spectrum (plotted in the insert of Fig. 3) clearly shows a series of integer harmonics $(2 f, 3 f, 4 f)$ and three extra peaks of subharmonics $(f / 2,3 f / 2$ and $5 f / 4)$. There is also excess power in the 
Table 1. Calculated periods, frequencies, amplitudes, phases, their uncertainties, and signal-to-noise ratios of the AAVSO and Kepler light curves.

\begin{tabular}{|c|c|c|c|c|c|c|c|c|c|}
\hline & ID & $P$ (day) & Freq. $(c / d)$ & amp (mag) & $\phi(\mathrm{rad} / 2 \pi)$ & \pm freq. $(c / d)$ & $\pm \operatorname{amp}(\mathrm{mag})$ & $\pm \phi(\mathrm{rad} / 2 \pi)$ & $S / N$ \\
\hline AAVSO & $f_{\mathrm{L}}$ & 779.606 & 0.0012827 & 1.1427 & 0.3710 & $2.57 \mathrm{e}-07$ & $9.09 \mathrm{e}-03$ & $1.27 \mathrm{e}-03$ & 67.48 \\
\hline- & $f$ & 24.916 & 0.0401350 & 0.2389 & 0.2138 & $1.23 \mathrm{e}-06$ & $9.09 \mathrm{e}-03$ & $6.06 e-03$ & 19.03 \\
\hline- & $\sim f / 2$ & 50.099 & 0.0199604 & 0.0819 & 0.5890 & $3.58 \mathrm{e}-06$ & $9.09 \mathrm{e}-03$ & $1.77 \mathrm{e}-02$ & 5.31 \\
\hline Kepler & $f_{\mathrm{L}}$ & 785.824 & 0.00127255 & 1.30205 & 0.417638 & $1.84 \mathrm{e}-08$ & $6.40 \mathrm{e}-05$ & $7.83 e-06$ & 3580.48 \\
\hline- & $f$ & 24.925 & 0.04012110 & 0.39100 & 0.821511 & $6.14 \mathrm{e}-08$ & $6.40 \mathrm{e}-05$ & $2.61 \mathrm{e}-05$ & 870.38 \\
\hline- & $f / 2$ & 49.915 & 0.02003410 & 0.08026 & 0.539616 & $2.99 \mathrm{e}-07$ & $6.40 \mathrm{e}-05$ & $1.27 \mathrm{e}-04$ & 211.68 \\
\hline- & $2 f$ & 12.460 & 0.08025420 & 0.08660 & 0.593535 & $2.77 \mathrm{e}-07$ & $6.40 \mathrm{e}-05$ & $1.18 \mathrm{e}-04$ & 171.36 \\
\hline- & $3 / 2 f$ & 16.634 & 0.06011710 & 0.04373 & 0.061574 & $5.49 \mathrm{e}-07$ & $6.40 \mathrm{e}-05$ & $2.33 \mathrm{e}-04$ & 107.01 \\
\hline- & $f / 4$ & 96.109 & 0.01040490 & 0.03819 & 0.370853 & $6.29 \mathrm{e}-07$ & $6.40 \mathrm{e}-05$ & $2.67 e-04$ & 108.37 \\
\hline- & $f / 3$ & 74.454 & 0.01343110 & 0.02637 & 0.627438 & $9.11 \mathrm{e}-07$ & $6.40 \mathrm{e}-05$ & $3.87 \mathrm{e}-04$ & 74.11 \\
\hline- & $3 f$ & 8.308 & 0.12037000 & 0.02669 & 0.243770 & $9.00 \mathrm{e}-07$ & $6.40 \mathrm{e}-05$ & $3.82 \mathrm{e}-04$ & 37.45 \\
\hline- & $5 / 4 f$ & 19.925 & 0.05018830 & 0.01189 & 0.196658 & $2.02 \mathrm{e}-06$ & $6.40 \mathrm{e}-05$ & $8.57 \mathrm{e}-04$ & 26.23 \\
\hline- & $4 f$ & 6.235 & 0.16039200 & 0.01090 & 0.162611 & $2.20 \mathrm{e}-06$ & $6.40 \mathrm{e}-05$ & $9.35 \mathrm{e}-04$ & 12.05 \\
\hline- & $5 / 2 f$ & 9.986 & 0.10013900 & 0.01126 & 0.927556 & $2.13 \mathrm{e}-06$ & $6.40 \mathrm{e}-05$ & $9.06 \mathrm{e}-04$ & 19.06 \\
\hline- & $7 / 2 f$ & 7.083 & 0.14118000 & 0.00369 & 0.488105 & $6.51 \mathrm{e}-06$ & $6.40 \mathrm{e}-05$ & $2.76 \mathrm{e}-03$ & 4.85 \\
\hline- & $5 f$ & 5.012 & 0.19952900 & 0.00374 & 0.003264 & $6.41 \mathrm{e}-06$ & $6.40 \mathrm{e}-05$ & $2.72 \mathrm{e}-03$ & 4.48 \\
\hline- & $6 f$ & 4.172 & 0.23969100 & 0.00199 & 0.033615 & $1.21 \mathrm{e}-05$ & $6.40 \mathrm{e}-05$ & $5.12 \mathrm{e}-03$ & 3.30 \\
\hline
\end{tabular}

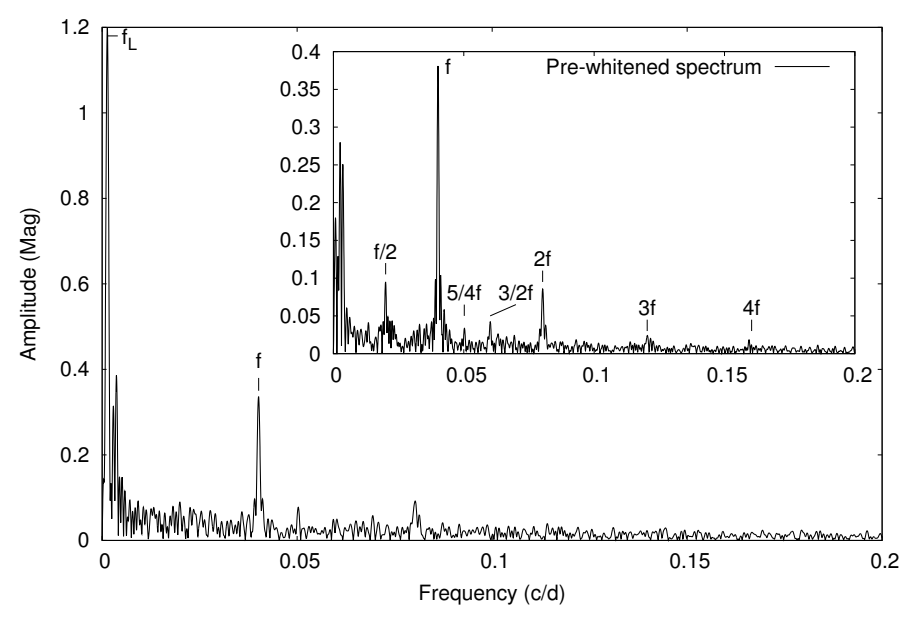

Fig. 3. Frequency spectrum of the Kepler data. The residual spectrum after pre-whitening with $f_{\mathrm{L}}$ is plotted in the insert.

low-frequency range, which is caused by the unstable shape of the two long-period cycles. Subsequent pre-whitenings resulted in clear detections of further harmonics and subharmonics, up to $6 f$ and $7 f / 2$ (see the full list in Table 1). The procedure was stopped when the amplitude of the highest peak (signal) was below the triple value of the mean amplitude around that peak (noise), so that the signal-to-noise ratio was below 3.0. It is well worth noting that no frequency independent of $f_{\mathrm{L}}$ or $f$ was found in the analysis.

To analyse the short-period variability separately, we have decomposed the Kepler light curve into two components. The smooth, long-term variation was approximated by fitting a polynomial function that was not sensitive to the fast fluctuations due to the pulsations. This has resulted in a somewhat better residual than pre-whitening with low-frequency sine waves, which were unable to follow the flat-topped maxima of the long-period variation. The components of the Kepler light curve are shown in the two panels of Fig. 4, where it is very apparent that the amplitude of the short-period variability changed seemingly randomly by a factor of 2 during the more than four years of observations.

We plot the phase diagram of the short-term residual variation in Fig. 5. This graph was made using a period of 49.99 days,

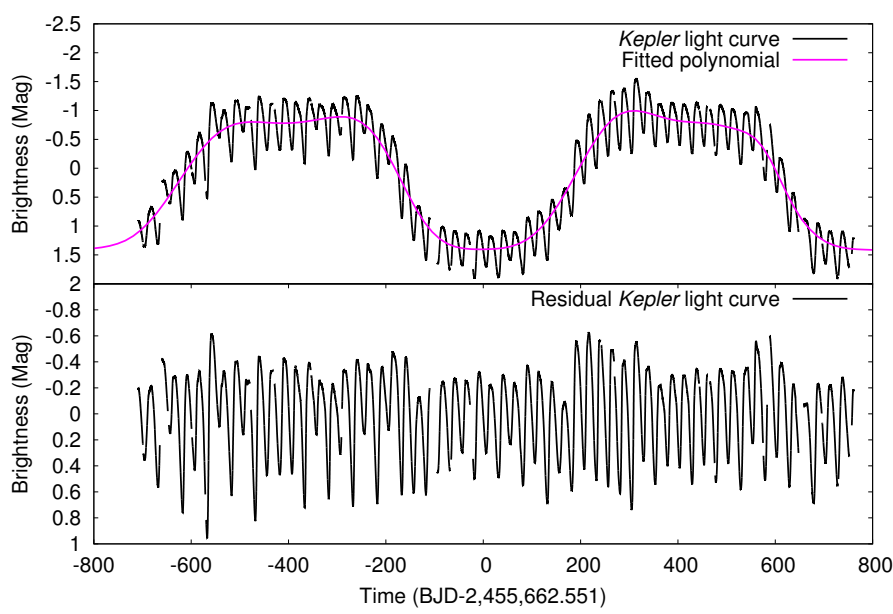

Fig. 4. Top: original Kepler light curve (black) and the fitted smooth polynomial (magenta). Bottom: the residual Kepler light curve after the subtraction of the long-term variation. The time axis is centred on the long-term minimum.

which is a refined pulsation period from the $\mathrm{O}-\mathrm{C}$ analysis (see below). While the alternating minima are obvious, it is also visible that the ranges of the maxima and the minima are similarly broad, so that the amplitude variations are not restricted to any special phases of the pulsation cycles. Both ascending branches from the two kinds of minima are steeper than the descending branches after the consecutive maxima. The overall light curve shape is similar to that of the Cepheid-like pulsators.

Part of the spread in Fig. 5 is caused by variations in phase that can be revealed by the traditional method of the $\mathrm{O}-\mathrm{C}$ diagram. This is a plot of the differences between the times of observed and calculated minima or maxima as a function of time. This approach is useful when the light curve is long enough and over many cycles the tiny changes accumulate to a detectable shift in phase. For DF Cygni, the AAVSO data were not suitable for an $\mathrm{O}-\mathrm{C}$ analysis because of the scarcity of the fiveday binned light curves, which prevented a detailed analysis of individual pulsation cycles. On the other hand, the accuracy, sampling rate, and continuity of the Kepler data permitted an 


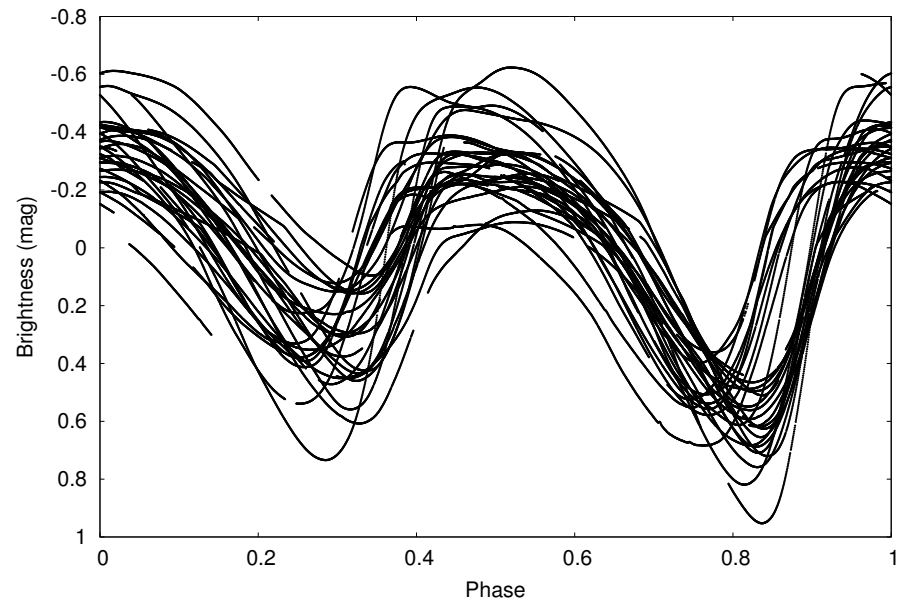

Fig. 5. Phase diagram of the residual Kepler light curve with a period of 49.99 days after subtraction of the long-term polynomial.

accurate determination of the individual times of minima for each pulsation cycle. However, a basic assumption of the $\mathrm{O}-\mathrm{C}$ analysis is the constancy of the light curve shape, i.e. any change in period or phase is just a tiny perturbation to the overall variation. This is not the case for DF Cygni, but we still feel that there is some value in the method.

To measure the times of minimum we fitted the light curves in a narrow range (1-3 days) of the minima with the following log-normal function:

$f(t)=\alpha \times \exp \left(-\ln (2) \times\left(\frac{\ln \left(2 \times \gamma \times \frac{(t-O)}{\beta}+1\right)}{\gamma}\right)^{2}\right)+c$

where $\alpha, \beta, \gamma$, and $c$ are constant parameters, and $O$ is the observed minimum time that is also obtained by the fitting procedure. The choice of the function was driven by the asymmetry around the minimum and the small number of parameters. The fitted parameters and their uncertainties were calculated using the $\chi^{2}$ method. The typical uncertainty of $O$ is approximately $\pm 0.0037 \mathrm{~d}$, which corresponds to $5.3 \mathrm{~min}$, that is about $1 / 6$ th of the Kepler long-cadence sampling. As usual in the $\mathrm{O}-\mathrm{C}$ analyses of RV Tau-type variables (Percy et al. 1997), we used the epochs of minima because of the sharper shapes around the minimum brightnesses.

We adopted the following ephemeris for the O-C plot: $T_{0}=$ BJD 2454994.58 and $P=49.99 \mathrm{~d}$. The period value was chosen in such a way that it leads to the flattest $\mathrm{O}-\mathrm{C}$ plot, predominantly centred on 0 . What we found from the Kepler data, however, is that the points in the $\mathrm{O}-\mathrm{C}$ diagram are actually split into two branches with a sharp transition between the two (see Fig. 6). In roughly 100 days between BJD $=2455900$ and 2456000 , there was an apparent phase shift of about 3 days (6\% of the pulsation period), after which the pulsation period remained the same. As revealed by the time-frequency analysis, the phase shift coincided exactly with the largest amplitude state of the pulsation, an episode during which the pulsation amplitude was about twice as high as immediately before and after. The very first point in Fig. 6 is a similarly down-scattered value that matches the phase of the data after the phase jump 1000 days later. On the other hand, the cycle-to-cycle scatter is well above the measurement error, which is smaller than the symbol size in Fig. 6.

To uncover finer details of the changes in amplitude and frequency content as function of time, we calculated the wavelet

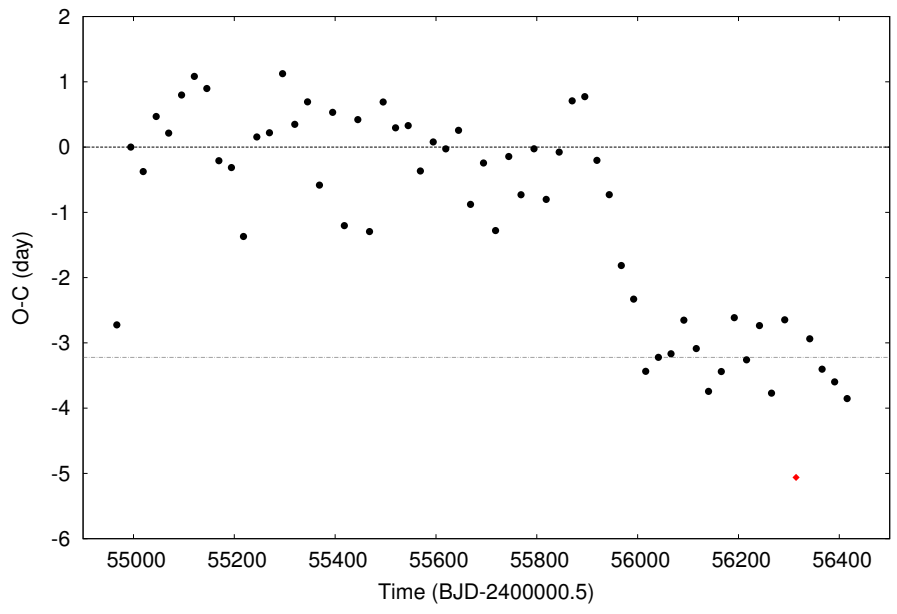

Fig. 6. O-C diagram of the Kepler light curve with $T_{0}=54994.58$ and $P=49.99$ days. The horizontal lines are the mean values of the group of points.
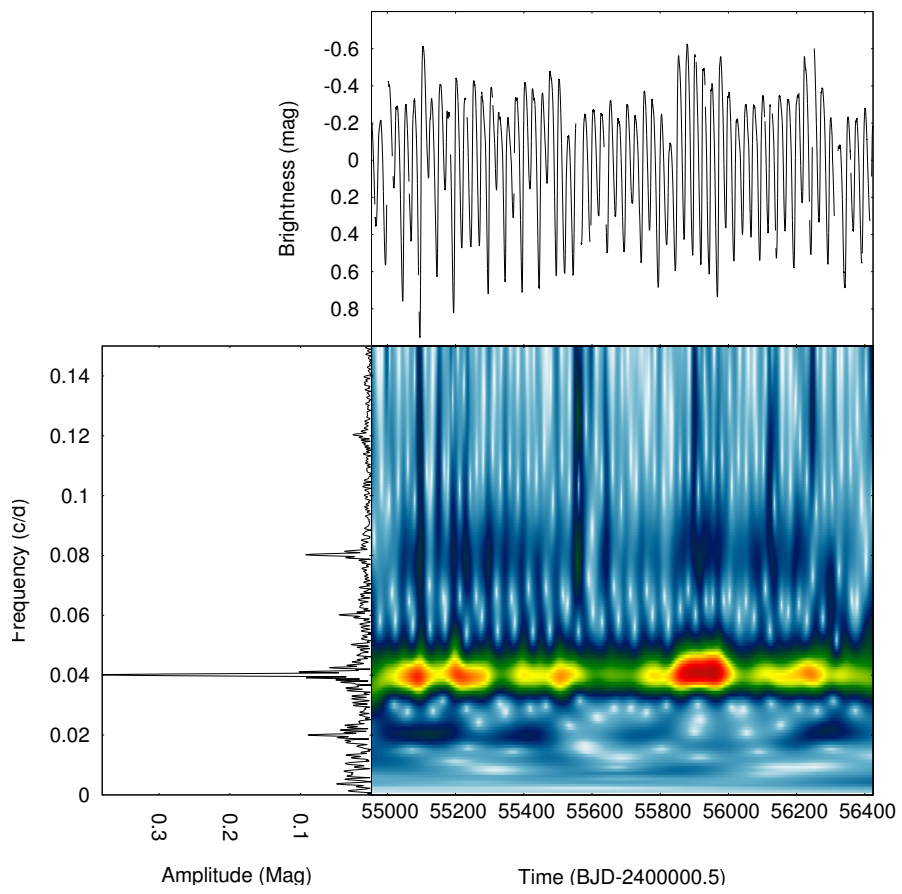

Fig. 7. Top: residual light curve after subtracting the slow variability; bottom: the frequency spectrum and wavelet map.

map in which the most prominent feature is the amplitude ridge of the frequency $f$ (see Fig. 7). In addition, the integer and halfinteger multiples are also recognizable. For the sake of convenience we plotted the light curve on the top of the map and the Fourier-spectrum on the left-hand side. The colour encoding represents the variations in amplitude, both in time and frequency. It is very apparent that the amplitudes of all peaks have changed tremendously, whereas the relative differences in the changes of the harmonics indicate significant variations in the overall shape of the light curve.

To get a more quantitative picture of the amplitude variations, we summed the peaks of the ridges of the first three strongest, well-separated frequencies in the wavelet map. For this we traced the peaks of the ridges to measure the instantaneous amplitude of each frequency. Their sum is a quantitative measure of the full oscillation amplitude; the role of the 


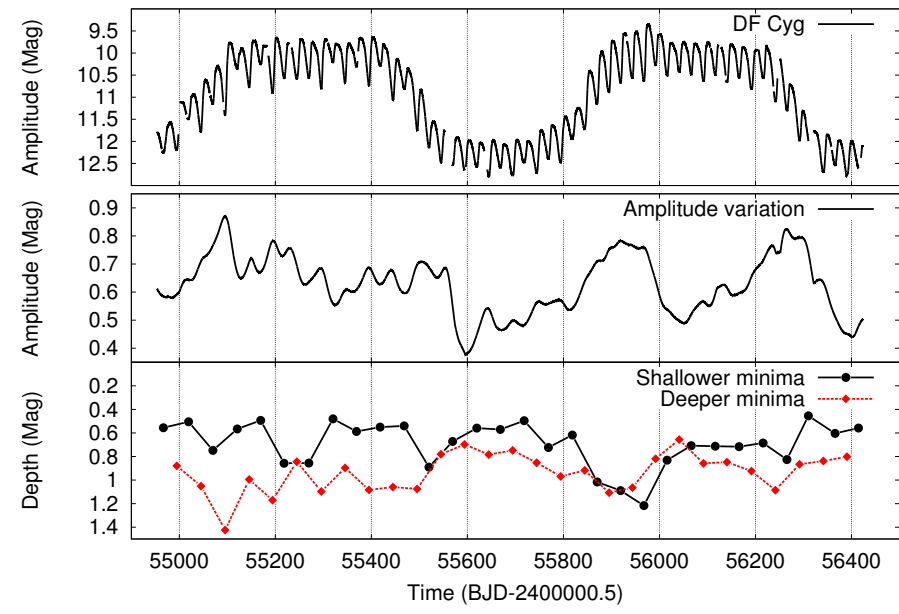

Fig. 8. Top: original Kepler light curve for comparison. Middle: the amplitude variation of the sum of the $0.02,0.04$ and $0.08 \mathrm{c} / d$ frequencies. Bottom: the variation of the depth of the shallower (black dots) and deeper minima (red diamonds).

phase differences is neglected this way. The result is shown in the middle panel of Fig. 8. There seems to be a correlation between the phase of the RVb cycle and the summed amplitude variation. The latter is smallest when the light curve is in deep minima (in the middle at BJD 2455600 and at the end, at BJD 2 456400). Furthermore, the summed amplitude also displays a sudden decrease right after the second phase jump (at about BJD 2456000). Overall, there is a slight indication that the total amplitude of pulsation is somewhat sensitive to the actual mean brightness, i.e. the pulsation is not entirely coupled from the long-period variation.

We also examined the variations of the depths of both types of minima. This was carried out by subtracting the mean brightness of the preceding and following maxima from the brightness of each minimum. The result is presented in the bottom panel of Fig. 8. The two kind of depths are plotted separately with the assumption that the two minima follow a regular alternating pattern. The plot reveals that throughout the whole Kepler observations the "deeper" minimum indeed almost always had a greater depth, except a short transitory phase from BJD 2455900 to 2456000 . Interestingly, this behaviour exactly coincides with the $\sim 100 \mathrm{~d}$ period when the peak-to-peak amplitude had a maximum and when the phase shift of about three days occured.

\section{Discussion}

How typical an RV Tau-type star is DF Cygni? To shed light on the answer, we plotted the location of DF Cygni in the $V$-band amplitude versus effective temperature and the luminosity versus effective temperature diagrams of selected post-AGB variables studied by Kiss et al. (2007). For the effective temperature we adopted $T_{\text {eff }}=4840 \mathrm{~K}$ (Brown et al. 2011; Giridhar et al. 2005), while the $V$-band amplitude was approximated by the mean peak-to-peak amplitude of the phase diagram in Fig. 5, which is about $1.0 \pm 0.2 \mathrm{mag}$. The luminosity has been estimated using the RV Tauri period-luminosity relation in the Large Magellanic Cloud, following the same approach as Kiss et al. (2007). The estimated luminosity is $1200 \pm 300 L_{\odot}$, where the uncertainty reflects the standard deviation of the LMC P-L relation. In the top panel of Fig. 9, DF Cygni falls close to several high-amplitude stars, which are all typical RV Tau-type objects (such as U Mon and AI Sco, both in the RVb class). Similarly
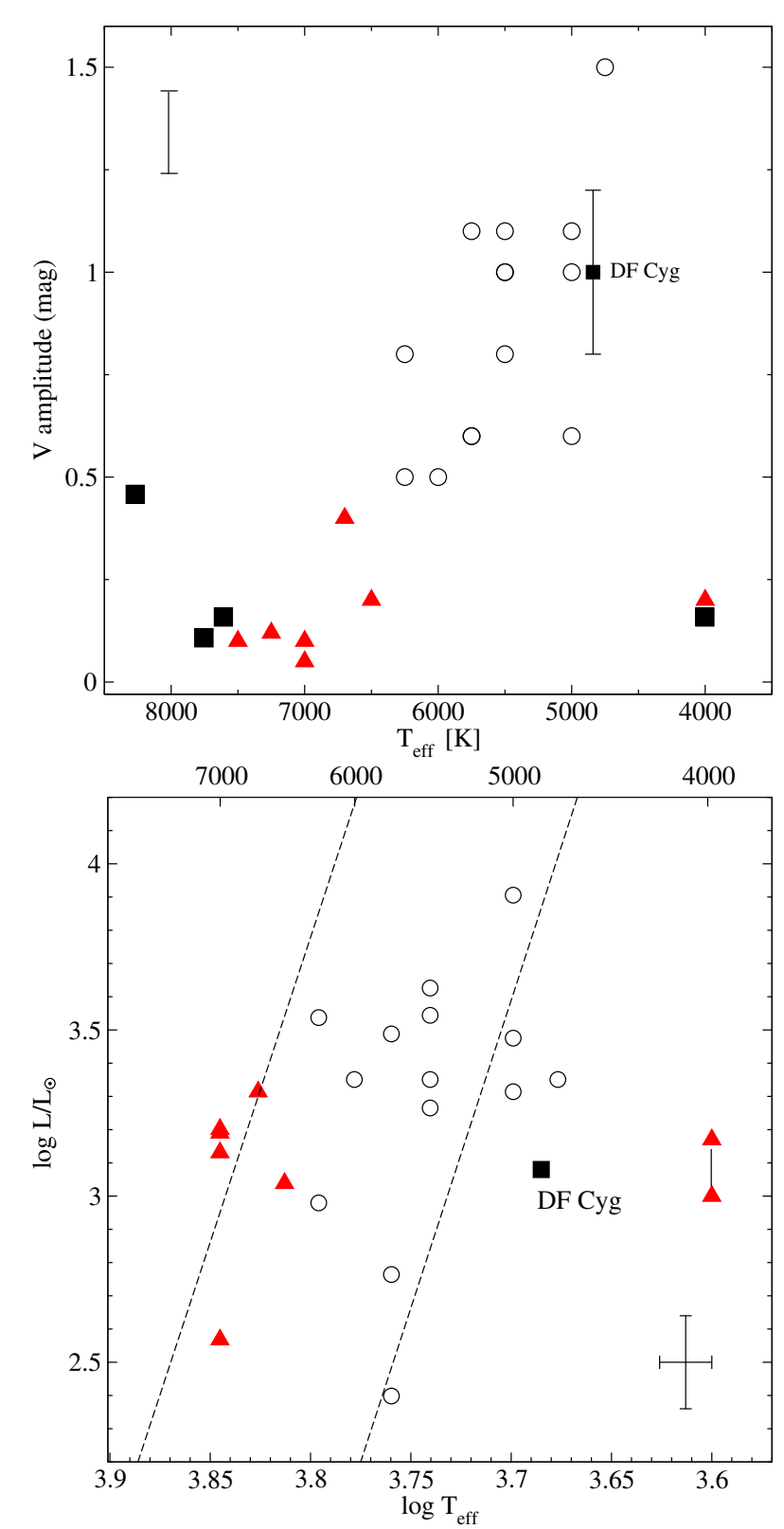

Fig. 9. Top panel: $V$-band amplitude as a function of effective temperature for the Kiss et al. (2007) sample of post-AGB variables, supplemented with the location of DF Cygni. Open circles: single periodic stars; triangles: multiperiodic/semi-regular stars; squares: variability due to orbital motion. The bar in the upper left corner shows the typical cycle-to-cycle amplitude variation in the pulsating stars. Bottom panel: the empirical HRD of the pulsating sample of Kiss et al. (2007) and the location of DF Cygni. The dashed lines show the edges of the classical instability strip, taken from Christensen-Dalsgaard (2003). The error bars in the lower right corner represent $\pm 3 \%$ error in effective temperature (about $200 \mathrm{~K}$ in the range shown) and the \pm 0.35 mag standard deviation of the LMC P-L relation.

good agreement is found in the empirical Hertzsprung-Russell diagram in the bottom panel of Fig. 9. Although DF Cygni lies somewhat beyond the red edge of the classical instability strip, and its location is consistent with its classification. Hence its light curve properties are likely to be representative for the whole class.

DF Cygni shows a very rich behaviour on all timescales. The slow variation has a period of $779.606 \mathrm{~d}$ and it has been remarkably coherent during the whole $48 \mathrm{yr}$ of visual observations. On 
top of the long-term cycles the pulsations appear with a period of $24.925 \mathrm{~d}$ (or the double period of $49.85 \mathrm{~d}$ if we take the RV Tau-type alternation of the cycles into account). Both types of light variation significantly fluctuate in time with a constantly changing interplay of amplitude and phase modulations.

In the Fourier-spectrum of the Kepler dataset, a characteristic series of subharmonics of the frequency $f$ appears, which is often interpreted as sign of period-doubling associated with an underlying low-dimensional chaotic behaviour. Similar phenomena were detected in several long-period pulsating variable stars, such as the arch-type of chaotic stars, the RV Tautype R Sct (Buchler et al. 1995, 1996), the less irregular RVa star AC Her (Kolláth et al. 1998), several semi-regular variables (Buchler et al. 2004), and one Mira-type variable (Kiss \& Szatmáry 2002). More recently, Kepler has opened a whole new avenue of RR Lyrae studies based on the period-doubling phenomenon and related effects (e.g. Szabó et al. 2010; Plachy et al. 2013, 2014; Benkő et al. 2014; Moskalik et al. 2015). Considering RV Tau-type variability, there is a strong need for more theoretical investigations. The only recent study that touches the domain in which DF Cygni resides is that of Smolec (2016), who studied a grid of non-linear convective Type II Cepheid models. Although his study does not cover the full parameter range of RV Tau-type stars, some of the models extend close to the temperature and luminosity of DF Cygni. For example, Smolec presented a detailed discussion of the resonances, which affect the shape of the model light curves, and in his Fig. 13 one can see the regions of the 2:1 resonances of the fundamental, first, and second overtone modes. The corresponding Fourier parameters are shown in Figs. 14, 15 of Smolec (2016), where DF Cygni's location is just outside at the top of the high-luminosity edge of the more massive model calculations. Here, near the red edge of the instability strip, the resonance between the fundamental mode and the first overtone is dominant (see the V-shaped dark features in Figs. 13-15, in the rightmost panels with the $M=0.8 M_{\odot}$ models). The observed complexity of the DF Cygni pulsations is consistent with the strongly non-linear behaviour of the models.

As we have seen in Sect. 3, all the periods, amplitudes, and phases vary in time, and none of these variations is strictly periodic. As has been revealed by the $\mathrm{O}-\mathrm{C}$ diagram, there was a transient episode in the light curve around BJD 2456000 , where the change of the period and the amplitude was remarkable. Right after the ascending branch of the RVb cycle, the amplitude of the pulsation increased by almost a factor of two, whereas the period decreased dramatically (approximately by 3-4 percent, that is about 1 day per cycle). After four to six cycles, the pulsation returned to the previous state. The fact that the sudden amplitude increase is associated with a period decrease is surprising, given that the typical period-amplitude correlation of classical pulsating stars is just the opposite. Interestingly, Smolec (2016) studied the differences between the linear and non-linear pulsation models and found that the relative difference between the non-linear and the linear periods can be up to $15 \%$, with a strong dependence in the sign across the instability strip. We find a striking feature in Fig. 6 of Smolec (2016): models near the location of DF Cygni show a strong and negative period difference, meaning that the non-linear models have periods that are shorter than those of the linear calculations. We may speculate that it is not a coincidence that DF Cygni's location and the region where the non-linear pulsation period is significantly smaller agree so well. If the high-amplitude-short-period transient of the light curve is caused by the pulsations becoming more non-linear then the Smolec (2016) models offer a natural explanation to the observed phenomenon. So that the sudden change in the light curve characteristics perhaps can be explained by the emergence of non-linear effects.

Both the variations of the phase diagram and the point-topoint scatter of the $\mathrm{O}-\mathrm{C}$ diagram (Figs. 5, 6) are much unlike the stability of the Cepheid-type variables. However, recent space observations of Type I Cepheids have found small cycle-to-cycle fluctuations (Derekas et al. 2012), which depend on the mode of pulsation, in which the first overtone is less stable than the fundamental mode (Evans et al. 2015). Although Type II Cepheids have not yet been covered extensively by space data, one can imagine the outlines of a progression of becoming more unstable on the timescale of the pulsations as we move closer to the non-linear regime of RV Tau-type stars.

Besides the properties of the pulsations, the nature of the $\mathrm{RVb}$-phenomenon, the long-term change of the mean brightness is also worth some discussion. The most common explanation of the RVb-phenomenon is a binary system that is periodically obscured by a circumstellar or a circumbinary dust disk (Waelkens \& Waters 1993; Pollard et al. 1996, 1997; Van Winckel et al. 1999; Maas et al. 2002), although some of the observed characteristics were difficult to reconcile with the dusty disk model (Pollard et al. 1996, 1997). The most recent infrared results from the WISE satellite (Gezer et al. 2015) indicate that RVb stars are exclusively those objects that have well-detected circumstellar disks, while there is also a clear correlation between disk sources and binarity. The 48-year coherence of the long-period variability of DF Cygni is indeed indicative of a stable mechanisms such as binary motion.

We find noteworthy the similarities between the RVb-type variability and the long secondary periods (LSPs) of red giant stars, the latter still representing a mystery (Nicholls et al. 2009, and references therein; Soszynski \& Wood 2013; Saio et al. 2015; Takayama et al. 2015). Among the suggested solutions we find binarity, variable extinction in circumstellar disks, or strange non-radial oscillations, many of these solutions somewhat resembling the proposed explanations of the RVb phenomenon. LSP-like variations have also been found in pulsating red supergiants (Kiss et al. 2006; Yang \& Jiang 2012), suggesting that the phenomenon may not be restricted to red giants. We find some correlations between the pulsations and the RVb-cycle; most notably the amplitude of the pulsations tends to be smaller in the faint states of DF Cygni, although the case is not entirely clear. Interestingly, van Aarle et al. (2011) cross-correlated their sample of candidate post-AGB stars with long period variables (LPV) from MACHO. They found 245 variables falling on the distinct period-luminosity relation of the long secondary periods. While some of those stars may not be genuine post-AGB objects, it is an interesting question if there is some connection between the LSPs of red giants and post-AGB variability. As has been pointed out by the referee, with what we know today, it seems most logical to conjecture that these red giants with long cycles are also binaries; but these binaries have longer orbital periods than RV Tauri stars, so that the same mass-transfer phenomena occurred later in the evolution of the primary, which could climb further on the AGB.

\section{Summary}

The main results of this paper can be summarized as follows:

1. We have combined almost $50 \mathrm{yr}$ of visual observations from the AAVSO and about four years of Kepler data to perform the most detailed light curve analysis of an RV Tau-type variable star ever obtained. 
2. The bright RVb-star DF Cygni is a typical member of the class, showing a prominent long-period mean brightness change and Cepheid-like pulsations that change constantly in amplitude and phase.

3. The Fourier spectrum of the Kepler data indicate the presence of a complex period-doubling pattern, revealed by the characteristic set of subharmonics. The time-frequency distributions clearly show the non-repetitive variations of the amplitudes.

4. The Kepler light curve also displays some transients, most notably one at BJD 2456000 , during which the anticorrelated period and amplitude variations could be an indication of emerging non-linear effects.

5. The long-term coherence of the RVb modulation is consistent with binary motion, and we note some similarities with the long secondary periods of the pulsating red giants.

Acknowledgements. This project has been supported by the Hungarian National Research, Development and Innovation Office (NKFIH) grants K-104607 and $\mathrm{K}-115709$. The research leading to these results has received funding from the European Community's Seventh Framework Programme (FP7/2007-2013) under grant agreement No. 312844 (SPACEINN) and the ESA PECS Contract Nos. 4000110889/14/NL/NDe and 4000109997/13/NL/KML. We thank the referee, Prof. Christoffel Waelkens, for detailed comments that yielded significant improvement to the paper. L.L.K. thanks the hospitality of the Veszprém Regional Centre of the Hungarian Academy of Sciences (MTA VEAB), where part of this project was carried out. The Kepler Team and the Kepler Guest Observer Office are recognized for helping to make the mission and these data possible. We acknowledge with thanks the variable star observations from the AAVSO International Database contributed by observers worldwide and used in this research. Fruitful discussions with Drs. R. Szabó and E. Plachy are gratefully acknowledged.

\section{References}

Bányai, E., Kiss, L. L., Bedding, T. R., et al. 2013, MNRAS, 436, 1576 Benkő, J., Plachy, E., Szabó, R., Molnár, L., \& Kolláth, Z. 2014, ApJS, 213, 31 Brown, T. M., Latham, D. W., Everett, M. E., \& Esquerdo, G. A. 2011, AJ, 142, 112

Buchler, J. R., Serre, T., \& Kolláth, Z. 1995, Phys. Rev. Lett., 74, 842

Buchler, J. R., Kolláth, Z., Serre, T., \& Mattei, J. 1996, ApJ, 462, 489

Buchler, J. R., Kolláth, Z., \& Cadmus, R. R. 2004, ApJ, 613, 532

Christensen-Dalsgaard, J., 2003, Lecture Notes on Stellar Oscillations, Aarhus University, May 2003, Fifth edition, http://astro.phys.au.dk/ jcd/ oscilnotes

Derekas, A., Szabó, Gy. M., Berdnikov, L., et al. 2012, MNRAS, 425, 1312

Evans, N. R., Szabó, R., Derekas, A., et al. 2015, MNRAS, 446, 4008

Fokin, A. B., 2001, Stellar Pulsation-Nonlinear Studies, eds. M. Takeuti, \& D. D. Sasselov, Astrophys. Space Sci. Libr., 103
Foster, G. 1996, AJ, 112, 1709

Gezer, I., Van Winckel, H., Bozkurt, Z., et al. 2015, MNRAS, 453, 133

Giridhar, S., Lambert, D. L., Reddy, B. E., Gonzalez, G., \& Yong, D. 2005, ApJ, 627,432

Harwood, M. 1927, Harvard College Observatory Bulletin, 847, 5

Harwood, M. 1936, Publications of the American Astronomical Society, 8, 10

Harwood, M. 1937, Annals of the Astronomical Observatory of Harvard College, 105,521

Jura, M. 1986, ApJ, 309, 732

Kiss, L. L., \& Szatmáry, K. 2002, A\&A, 390, 585

Kiss, L. L., Szatmáry, K., Cadmus, R. R. Jr., \& Mattei, J. A. 1999, A\&A, 346, 542

Kiss, L. L., Szabó, Gy. M., \& Bedding, T. R. 2006, MNRAS, 372, 1721

Kiss, L. L., Derekas, A., Szabó, Gy. M., Bedding, T. R., \& Szabados, L. 2007, MNRAS, 375, 1338

Kolláth, Z. 1990, MNRAS, 247, 377

Kolláth, Z., Buchler, J. R., Serre, T., \& Mattei, J. 1998, A\&A, 329, 147

Lenz, P., \& Breger, M. 2005, Commun. Asteroseismol., 146, 53

Lloyd Evans, T. 1985, MNRAS, 217, 493

Maas, T., Van Winckel, H., \& Waelkens, C. 2002, A\&A, 386, 504

Moskalik, P., Smolec, R., Kolenberg, K., et al. 2015, MNRAS, 447, 2348

Nicholls, C. P., Wood, P. R., Cioni, M.-R. L., \& Soszynski, I. 2009, MNRAS, 399, 2063

Percy, J. R. 1993, in Luminous High-Latitude Stars, ed. D. D. Sasselov (San Francisco: ASP), 45, 295

Percy, J. R. 2006, JAAVSO, 34, 125

Percy, J. R., Bezuhly, M., Milanowski, M., \& Zsoldos, E. 1997, PASP, 109, 264

Plachy, E., Kolláth, Z., \& Molnár, L. 2013, MNRAS, 433, 3590

Plachy, E., Benkő, J., Kolláth, Z., Molnár, L., \& Szabó, R. 2014, MNRAS, 445, 2810

Pollard, K. R., Cottrell, P. L., Kilmartin, P. M., \& Gilmore, A. C. 1996, MNRAS, 279, 949

Pollard, K. R., Cottrell, P. L., Lawson, W. A., Albrow, M. D., \& Tobin, W. 1997, MNRAS, 286, 1

Pollard, K. R., McSaveney, J. A., \& Cottrelll, P. L. 2006, Mem. Soc. Astron. It., 77, 527

Preston, G. W., Krzeminski, W., Smak, J., \& Williams, J. A. 1963, ApJ, 137, 401

Saio, H., Wood, P. R., Takayama, M., \& Ita, Y. 2015, MNRAS, 452, 3863

Smolec, R. 2016, MNRAS, 456, 3475

Szabó, R., Kolláth, Z., Molnár, L., et al. 2010, MNRAS, 409, 1244

Takayama, M., Wood, P. R., \& Ita, Y. 2015, MNRAS, 448, 464

van Aarle, E., van Winckel, H., Lloyd Evans, T., et al. 2011, A\&A, 530, A45

Van Winckel, H., Waelkens, C., Fernie, J. D., \& Waters, L. B. F. M. 1999, A\&A, 343, 202

Waelkens, C., \& Waters, L. B. F. M., 1993, in Luminous High-Latitude Stars, ed. D. D. Sasselov (San Francisco: ASP), 45, 219

Wallerstein, G. 2002, PASP, 114, 689

Willson, L. A., \& Templeton, M., 2009, in Stellar Pulsation: Challenges for Theory and Observation (Melville, NY: Amer. Inst. Physics), AIP Conf. Proc., 1170,113

Wood, P. R., Alcock, C. Allsman, R. A., et al. 1999, IAU Symp. 191, eds. T. Le Bertre, A. Lebre, \& C. Waelkens, 151

Yang, M., \& Jiang, B. W. 2012, ApJ, 754, 35 